\title{
ORIENTATION-BASED PAIRWISE COARSE REGISTRATION WITH MARKERLESS TERRESTRIAL LASER SCANS
}

\author{
S. N. Mohd Isa ${ }^{1, *}$ S. A. Abdul Shukor ${ }^{1}$, N. A. Rahim ${ }^{1}$, I. Maarof ${ }^{2}$, Z. R. Yahya ${ }^{3}$, A. Zakaria ${ }^{1}$, A. H. Abdullah ${ }^{1}$, R. Wong ${ }^{4}$ \\ ${ }^{1}$ School of Mechatronic Engineering, Universiti Malaysia Perlis, 02600 Arau, Perlis, Malaysia \\ ${ }^{2}$ Faculty of Architecture, Planning and Surveying, UiTM, 02600 Arau, Perlis, Malaysia \\ ${ }^{3}$ Institute of Engineering Mathematics, Universiti Malaysia Perlis, 02600 Arau, Perlis, Malaysia \\ ${ }^{4}$ Geodelta System Sdn. Bhd., 22, Jalan SS20/11, Damansara Utama, 47400 Petaling Jaya, Selangor, Malaysia
}

\begin{abstract}
KEY WORDS: Point Cloud, Terrestrial Laser Scanner, Pairwise Registration, 3D Keypoints, Heritage Building, Keypoint Extraction
\end{abstract}

\begin{abstract}
:
In this paper, pairwise coarse registration is presented using real world point cloud data obtained by terrestrial laser scanner and without information on reference marker on the scene. The challenge in the data is because of multi-scanning which caused large data size in millions of points due to limited range about the scene generated from side view. Furthermore, the data have a low percentage of overlapping between two scans, and the point cloud data were acquired from structures with geometrical symmetry which leads to minimal transformation during registration process. To process the data, 3D Harris keypoint is used and coarse registration is done by Iterative Closest Point (ICP). Different sampling methods were applied in order to evaluate processing time for further analysis on different voxel grid size. Then, Root Means Squared Error (RMSE) is used to determine the accuracy of the approach and to study its relation to relative orientation of scan by pairwise registration. The results show that the grid average downsampling method gives shorter processing time with reasonable RMSE in finding the exact scan pair. It can also be seen that grid step size is having an inverse relationship with downsampling points. This setting is used to test on smaller overlapping data set of other heritage building. Evaluation on relative orientation is studied from transformation parameter for both data set, where Data set I, which higher overlapping data gives better accuracy which may be due to the small distance between the two point clouds compared to Data set II.
\end{abstract}

\section{INTRODUCTION}

Remote sensing has gradually been used in many applications such as in documentation of building heritage preservation. At present, Terrestrial Laser Scanner (TLS) is preferred in this area due to its capability of acquiring details three-dimensional (3D) data in a short time (Baik, 2017)(Klapa, Mitka, \& Zygmunt, 2017). TLS captures the object and the scene information in millions 3D point cloud which able to ensure the details of the building surface and its surrounding are collected. However, due to the requirement of multi-station scanning of TLS scan data, limited range about the scene generated from side view, thus cause heterogeneous in data (Che \& Olsen, 2018). Hence, registration is crucial processing element attracted the interest among researchers in order to combine the entire single scan.

Consequently, the challenges in point cloud data processing could also be started as early as during the data acquisition process. Artificial marker usually involved as a reference to differentiate between scan data and provide orientation order for registration process (Tremblay \& Béland, 2018). This work has some disadvantages in taking considerable time for proper arrangement to make sure each scan is having suitable overlapping vision and prevent from constellation scan. The placed marker also needs to be maintained at same position along the scanning process. In addition, data from occluded marker needs to take out at the end of data processing in the final product. Since the scanning mechanism of TLS is based on distance between the platform and the targeted object,

\footnotetext{
* Corresponding author.

Email addresses: sitinurhashima.isa@gmail.com (S. N. Mohd Isa), shazmin@unimap.edu.my (S. A. Abdul Shukor)
}

registration of point cloud data can be solved by determining the transformation matrix, rotation and translation of the rigid body. Usually, the transformation was solved using an approach of coarse-to-fine registration. Researchers find out that the coarse registration is critical procedure to align the point cloud data that next affect the further analysis in fine registration ( $\mathrm{Li} \&$ Chen, 2018).

Generally, the method for coarse registration involve features based on point, line or surface. The feature from the raw scene inherited from parameters such as geometrical corner or difference in intensity. Points feature usually known as keypoint are the most popular extracted feature because it produces slightly high accuracy in coarse registration (Kobbelt \& Botsch, 2004). In this work, the point feature is used for pairwise coarse registration with real world challenging data in consequence of (i) large data size with millions of points, (ii) low overlapping between two scan (around $40 \%$ only) and (iii) symmetrical geometry in scanning data representing the targeted object, i.e. the heritage building.

Therefore, in this paper, a solution using markerless is presented for coarse registration from TLS point cloud data. 3D keypoint of 3D Harris is used for two data set specifically for heritage building preservation, as the data represents real, old, aged building scene which were collected for conservation. This study is aims to evaluate (i) influence of different sampling method (random, grid average, non-uniform grid sample) related to processing time, (ii) variable of voxel grid size $(0.001 \mathrm{~m}, 0.025 \mathrm{~m}$ and $0.05 \mathrm{~m})$ relation to accuracy of data and (iii) to study characteristic and orientation of scan effect in pairwise registration. 


\section{RELATED WORK}

Pairwise coarse registration contains two procedure, firstly detection of feature or keypoint and secondly aligning all those corresponding points. Here, transformation of the scan pair are critical aspect that involve suitable translation and rotation in local coordinate system (Maiseli, Gu, \& Gao, 2017).

The main idea to keypoint determination in a data is based on geometric feature that having particular characteristic such as normal vector or different in intensity (Barnea \& Filin, 2008). For example, normal feature was used for building extraction whereby it shows the specific direction of vector represent a corner of the surface (Du et al., 2017). Consequently, 3D Harris keypoint started to be introduced for point cloud 3D registration (Rusu, Blodow, \& Beetz, 2009) due to its simplicity and generally this kind of point feature are dominant throughout the scene (Sipiran \& Bustos, 2011)(P W Theiler, Wegner, \& Schindler, 2014).

Popular ICP algorithm usually used in initial alignment for coarse registration. Although the algorithm pursued good accuracy ( $\mathrm{Li} \&$ Chen, 2018), but the enhancement in this method using feature of keypoint has managed to increase the precision in registration between two point clouds (He, Liang, Yang, Li, \& He, 2017). Furthermore, markerless registration deal with issue of finding best pairing scan orientation. Study shows that relative orientation of scan pairs using geometrical feature based are more effective although the case of low overlapping points (Brenner, Dold, \& Ripperda, 2008)(Shukor \& Aminuddin, 2017). Therefore, utilizing keypoints for unknown orientation has managed to register point cloud automatically (Hansen, 2004)(Wang \& Claus, 2008)

In this paper, the markerless pairwise coarse registration using 3D Harris keypoint is proposed. The effect of relative orientation between two scans studied to obtain behaviour of transformation parameter and the success of pairwise coarse registration.

\section{METHODOLOGY}

Registration of point cloud is almost the same process as in image processing. Both are utilizing feature extraction and for 3D point cloud, keypoint is used (Barnea \& Filin, 2008). This work focused on the heritage building which represents the object of interest in the scene. Fig. 1 shows the point-based registration process which contains element as follows:

Two raw point cloud data was applied for downsampling using either grid average, random sampling or non-uniform grid sample method, to reduce the data while preserving the distribution of point cloud. 3D Harris keypoint is then extracted from normal vector of the point.

- $\quad$ After downsampling, a fixed point cloud is used as a source scan, then registered with moving point cloud as target using ICP algorithm. Here, the transformation matrix shows rotation and translation using quaternion orientation in order to get the output.

- Initial alignment by ICP coarse registration then merged as registered pairwise of point cloud and the accuracy is studied by the root means squared error, RMSE.

- Relative orientation in terms of transformation matrix is then evaluated regarding the success registration of point cloud shown from the plot of two point cloud data.

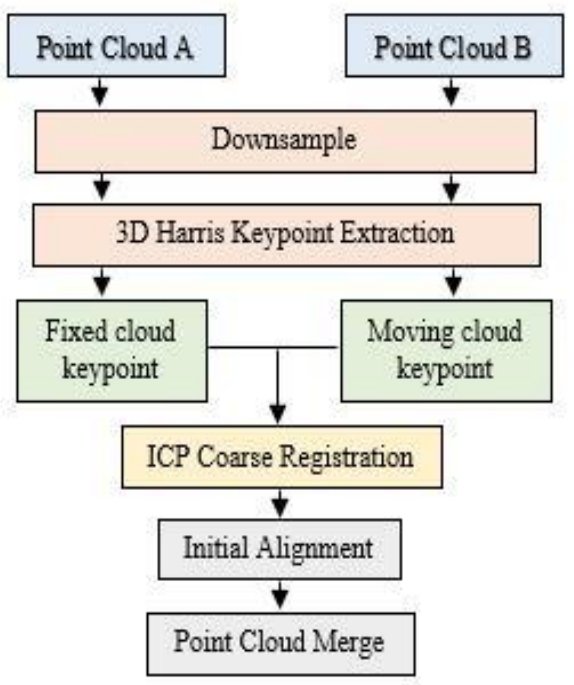

Figure 1. Block diagram of the method

Point cloud data from TLS can be registered automatically through finding of transformation matrix between corresponding point (Corina Daniela Păun, Valeria Ersilia Oniga, 2017). Relative orientation transform object to coordinate system by preserving their parameter of rotation angle and translation at $\mathrm{x}, \mathrm{y}, \mathrm{z}$ axes is given as Equation (1):

$$
\left[\begin{array}{l}
X \\
Y \\
Z
\end{array}\right]=\lambda R_{\mathrm{K} \Psi \omega}\left[\begin{array}{l}
X \\
Y \\
Z
\end{array}\right]+\left[\begin{array}{l}
T_{X} \\
T_{Y} \\
T_{Z}
\end{array}\right]
$$

Where $\quad\left[\begin{array}{lll}X & Y & Z\end{array}\right]^{T}=$ the coordinate of raw point cloud $\lambda=$ scalar matrix

$$
R_{\text {Кфш }}=\text { angle of rotation for } R_{\mathrm{Z}}(\kappa), R_{\mathrm{Y}}(\varphi),
$$

$R_{\mathrm{X}}(\omega)$ about $\mathrm{x}, \mathrm{y}, \mathrm{z}$ axes respectively

$\left[\begin{array}{lll}T_{X} & T_{Y} & T_{Z}\end{array}\right]^{T}=$ translation of point cloud at $\mathrm{x}$, $\mathrm{y}, \mathrm{z}$ axes respectively

The rotation matrix is orthogonal, thus condition for all points to match to their nearest neighbour is given by the scalar as in Equation (2):

$$
R^{T} R=I_{3} \quad \operatorname{det}(R)=+1
$$

In addition, $\mathrm{R}$ is calculated using $\mathrm{x}, \mathrm{y}, \mathrm{z}$ axes rotation angle $(\omega$, $\varphi, \kappa)$ respectively thus resulting as the following matrix form in Equation (3):

$\mathbb{R}=\left[\begin{array}{ccc}\cos \varphi \cos x & \sin \omega \sin \varphi \cos x-\cos \omega \sin x & \cos \omega \sin \varphi \cos x+\sin \omega \sin x \\ \cos \varphi \sin x & \sin \omega \sin \varphi \sin x+\cos \omega \cos x & \cos \omega \sin \varphi \sin x-\sin \omega \cos \mathrm{k} \\ -\sin \varphi & \sin \omega \cos \varphi & \cos \omega \cos \varphi\end{array}\right]$

Thus, the rotation angle from 3-dimension matrix can be determine by calculating using formulas shown in (4):

$\omega=\tan ^{-1}\left[\frac{r_{12}}{r_{10}}\right], \quad \varphi=-\sin ^{-1}\left[r_{31}\right], \quad \kappa=\tan ^{-1}\left[\frac{r_{21}}{r_{12}}\right]$ 


\section{KEYPOINT EXTRACTION}

The point cloud data by TLS with multi scan stations holds related information points between the scans. Reducing raw point cloud to keypoint for their corresponding between moving and fixed point cloud manage for lesser data and processing time. Moreover, keypoint could also increase the possibility in matching their correspondence point (Persad \& Armenakis, 2017).

Harris keypoint was implemented by (Rusu \& Cousins, 2011) for 3D data processing. This feature is preferred due to its simple application based on geometrical of local normal vector within nearest neighbour (Bueno, Martínez-Śanchez, GonźalezJorge, \& Lorenzo, 2016). In a way to extract keypoint, the raw point cloud was minimized using voxel grid sampling to achieve the regular array of data. This downsampling method using centroid is able to preserve actual representation of the point cloud. Fig. 2 shows the raw point cloud and its keypoint representative.

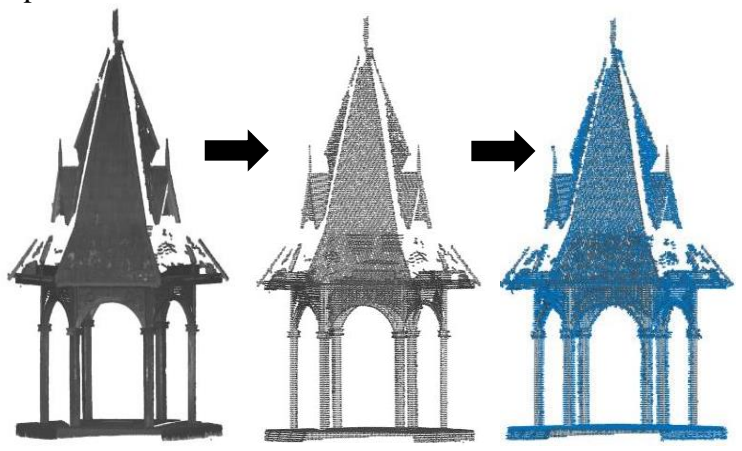

Figure 2. Keypoint extraction form raw data of point cloud, voxel grid downsample and 3D Harris keypoint

\section{EXPERIMENTS}

The parameters of the effect of different pairwise station is studied from orientation for a success registration. In this experiment, parameters of downsampling method and voxel grid size is evaluated using data set I (Belfry Bell Tower) and further implementation of good parameter for orientation study. By adopting the same setting parameter is continued for data set II (Tawau Lighthouse) for smaller overlapping case. The point cloud data representing the building is provided by Geodelta Systems Sdn. Bhd acquired using Leica HDS6000 TLS. The TLS is capable to scan up to 500,000 points per second and this system is equipped with a NIKON D80, 10.2 Megapixel camera.

The rigid transformation is performed in aligning the first point cloud (fixed) and second point cloud (moving) using ICP algorithm. The first point cloud used as the reference to estimate transformation to the raw second point cloud. Aligned point cloud then merged together by correspondence points based on nearest neighbour. Point-to-plane minimization error are applicable since obvious geometrical plane surface exist for a building (Grant, Bethel, \& Crawford, 2012). Moreover, the coarse registration of fixed and moving point cloud enforce convergence solution to reduce the number of iteration. The successful pairwise coarse registration directly define using converge ICP result by RMSE. All experiments were conducted using MATLAB R2018b and 64 Bits Windows 10 with Intel Core i5-4200U (1.60GHz) 12 GB RAM.

\subsection{Data set I (Belfry Bell Tower)}

Tawau Bell Tower or also known as the Belfry is in Tawau, Sabah, and was constructed as the evidence of previous British government before Sabah became part of Malaysia. The historic building is almost 100 years old and it is the oldest monument that was safe during the Second World War. The Bell Tower was built by prison labours to remember the resolution undersigned during First World War when Japan became cronies of England. This building was refurbished by Rotary Club of Tawau and passed to Jabatan Muzium Sabah on 2006 (Ali, 2016).

Fig. 3 shows the building structure of hexagon shaped. The challenge in this data set is due to its property of geometrical symmetry and low overlapping scanning points which is $\approx 40 \%$. The scanner located into 10 stations respectively around Bell Tower. Station one to station five (ST1 to ST5) and station six to station ten (ST6 to ST10) represents outdoor and indoor scan respectively as shown in Fig. 4. At first, ST1 and ST2 is used to evaluate downsample method and voxel grid size before moving to another combination of stations.

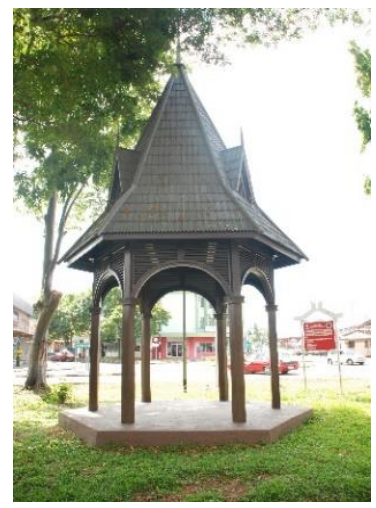

Figure 3. Bell tower

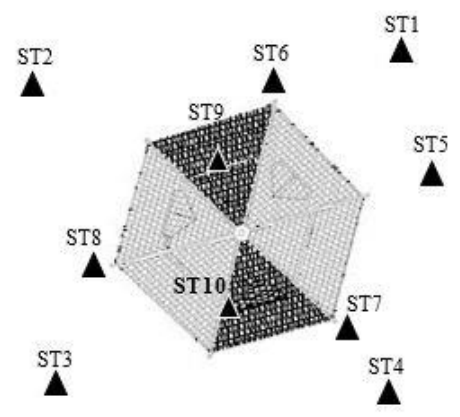

Figure 4. Scan location of Bell Tower 
Table 1. Evaluation of different sampling methods using ST1-ST2 scan pairs

\begin{tabular}{|c|c|c|c|c|}
\hline \multirow[b]{2}{*}{ Minimization error } & \multicolumn{2}{|c|}{ Downsample methods } & \multirow[b]{2}{*}{ Time (s) } & \multirow[b]{2}{*}{$\operatorname{RMSE}(\mathrm{m})$} \\
\hline & $\begin{array}{c}\text { Fixed cloud } \\
937985\end{array}$ & $\begin{array}{c}\text { Moving cloud } \\
1044604\end{array}$ & & \\
\hline \multirow{3}{*}{ Point-to-point } & Random & Random & \multirow{2}{*}{5189} & \multirow{2}{*}{0.128} \\
\hline & 937985 & 1044604 & & \\
\hline & $\begin{array}{c}\text { Grid average } \\
7214\end{array}$ & $\begin{array}{c}\text { Grid average } \\
7135\end{array}$ & 6 & 0.295 \\
\hline \multirow{2}{*}{ Point-to-plane } & $\begin{array}{c}\text { Grid average } \\
7214\end{array}$ & $\begin{array}{c}\text { Grid average } \\
7135\end{array}$ & 6 & 0.295 \\
\hline & $\begin{array}{c}\text { Random } \\
937985\end{array}$ & $\begin{array}{l}\text { Non-uniform grid sample } \\
131072\end{array}$ & 292 & 0.128 \\
\hline
\end{tabular}

Table 2. Grid average downsample with different grid size and different scans

\begin{tabular}{lrccc}
\hline \multirow{2}{*}{$\begin{array}{c}\text { Scan station } \\
\text { (points) }\end{array}$} & \multicolumn{3}{c}{$\begin{array}{c}\text { Grid average downsample points with } \\
\text { different grid size }\end{array}$} \\
\cline { 3 - 5 } & & $0.01 \mathrm{~m}$ & $0.025 \mathrm{~m}$ & $0.05 \mathrm{~m}$ \\
\hline ST1 & 937985 & 426137 & 95551 & 26250 \\
ST2 & 1044604 & 470731 & 100274 & 26829 \\
ST3 & 1257631 & 451498 & 89523 & 24162 \\
ST4 & 1025035 & 428315 & 86715 & 23166 \\
ST5 & 1139172 & 489343 & 101392 & 27382 \\
\hline
\end{tabular}

\subsection{Parameters selection}

The raw TLS point cloud was tested with different type of sampling method (random, grid average, non-uniform grid sample) in order to find the proper setting for downsampling the points. Comparison of those methods with respect to processing time and squared distance error of registration shows the accuracy of the respective method are shown in Table 1. ICP algorithm uses minimization error of given matrix (point-topoint or point-to-plane) to minimize the distance and estimate the transformation between fixed point cloud and moving point cloud. From the results, random sampling method using pointto-point and point-to-plane matrix has long processing time but same squared distance due to maximum return of downsampling point. Contrary, grid average downsample method results show the shortest processing time with almost two times squared distance error with respect to $0.1 \mathrm{~m}$ grid size. Thus, it is a consideration to use the grid average method which can divide irregular point cloud (P W Theiler, Wegner, \& Schindler, 2013) and point-to-plane matrix for better accuracy (Salvi, Matabosch, Fofi, \& Forest, 2007).

Voxel grid downsample works like a filter that able to reduce the number of points. In $3 \mathrm{D}$, the voxel grid divides the rectangular with respective given grid size. The points belong in a voxel will join into one output point. By taking the centroid of the voxel, it will show better accuracy because it considers distribution of point inside the voxel. This selection is suitable for uneven point cloud array due to computation average stored the normalization point in the first point cloud and remove the empty voxel without points (P W Theiler et al., 2013). Table 2 shows the relationship of grid average downsampling method with different grid size $(0.01 \mathrm{~m}, 0.025 \mathrm{~m}$ and $0.05 \mathrm{~m})$. The number of point cloud reduced with larger grid size. Thus, in this paper, grid size $0.05 \mathrm{~m}$ is applied for further evaluation. Some mentioned that there is no big influence of this parameter in registration accuracy (Xu, Boerner, Yao, Hoegner, \& Stilla,
2019). Therefore, proper registration can be done as long as there are sufficient of extracted information points (Pascal Willy Theiler, Wegner, \& Schindler, 2014).

\subsection{Evaluation on relative orientation}

To register two point clouds without knowledge on reference marker, manual evaluation on the effect of relative scan orientation are needed. Thus, this allow to study the pairwise coarse registration using scan pairs effect on overlapping points and accuracy based on squared distance, RMSE and transformation parameters. Different arrangement of scan station resulted in different overlapping point and effect on the registration error. Aligning the corresponding matching point required to encounter the orientation problem (Yamany, Farag, \& Member, 2002). Therefore, it is good to learn how certain software do the selection of station for registration purpose.

As a start, reference station using ST1 is randomly selected as the combination towards all scan station and vice versa as shown in Table 3. The results show registration with respect one station to the other nine stations give lower average squared distance, RMSE which higher in accuracy compared to the way combination nine stations to one station. In general, relationship of one-to-many orientation are relevant compared to many-toone orientation for this data set.

For outdoor scans, the best coarsely pair registration was ST1ST3 as seen in Fig. 5(a) which shows success of registration with lowest squared distance, RMSE. Having small value of transformation parameter, rotation and translation lead to proper registration (Brenner et al., 2008). Although, ST4-ST1 scan pair give highest overlapping percentage, but they are located far from each other scan, thus resulting in low accuracy with high squared distance, RMSE, as shown in Fig. 5(b). Meanwhile, for indoor scans, ST8-ST1 as seen in Fig. 5(c) shows high squared distance, RMSE while station ST1-ST9 in Fig. 5(d) managed to get good alignment even the percentage of overlapping point are the lowest. The lowest overlapping point enable coarsely register the point cloud using approach in this paper. Therefore, the success in pairwise registration are depending to distance between scan pairs (Wang \& Claus, 2008).

TLS data acquisition transform to world coordinate system on behalf of registration can be studied by attaining the transformation parameter, angle of rotation and translation. This approaches are common in remote sensing application. Object or point cloud scene propagate through their respective axis 
The International Archives of the Photogrammetry, Remote Sensing and Spatial Information Sciences, Volume XLII-4/W16, 2019 6th International Conference on Geomatics and Geospatial Technology (GGT 2019), 1-3 October 2019, Kuala Lumpur, Malaysia

Table 3. Scan pairs with transformation parameter, overlapping points and Root Means Square Error (RMSE). Results for data set I using point-to-plane minimization error and $0.05 \mathrm{~m}$ grid average downsampling method.

\begin{tabular}{|c|c|c|c|c|c|c|c|c|}
\hline Scan pairs & $\omega\left(^{\circ}\right)$ & $\varphi\left({ }^{\circ}\right)$ & $\kappa\left({ }^{\circ}\right)$ & Tx (m) & Ty $(\mathrm{m})$ & $\mathrm{Tz}(\mathrm{m})$ & Overlap (\%) & RMSE (m) \\
\hline ST1ST2 & -0.543 & -0.187 & -35.760 & 0.585 & 2.718 & -0.053 & 42.904 & 0.268 \\
\hline ST1ST3 & 2.197 & 0.510 & -2.626 & -2.317 & 0.277 & 0.050 & 38.686 & 0.118 \\
\hline ST1ST4 & -4.637 & -0.520 & 44.245 & -0.652 & 7.394 & 0.173 & 43.400 & 0.224 \\
\hline ST1ST5 & -3.086 & -1.260 & -50.455 & 0.367 & 3.320 & 0.065 & 41.957 & 0.144 \\
\hline ST1ST6 & 0.811 & -0.760 & -29.323 & -4.753 & 1.382 & 0.150 & 29.034 & 0.317 \\
\hline ST1ST7 & 2.144 & 0.769 & -34.770 & -4.805 & 1.519 & 0.131 & 28.324 & 0.242 \\
\hline ST1ST8 & 1.700 & 1.069 & -32.784 & -4.677 & 1.510 & 0.242 & 27.774 & 0.159 \\
\hline ST1ST9 & -3.105 & -3.395 & -8.264 & -6.662 & 4.922 & 0.555 & 25.347 & 0.317 \\
\hline ST1ST10 & -3.057 & -2.300 & -5.539 & -6.728 & 4.791 & 0.585 & 25.509 & 0.314 \\
\hline Average & -0.842 & -0.675 & -17.253 & -3.294 & 3.092 & 0.211 & 33.659 & 0.234 \\
\hline ST2ST1 & 0.064 & -1.076 & 33.344 & -1.820 & -1.758 & -0.025 & 43.919 & 0.211 \\
\hline ST3ST1 & -5.178 & -0.593 & 13.089 & 1.392 & -1.217 & -0.406 & 42.269 & 0.208 \\
\hline ST4ST1 & 3.988 & -11.644 & 51.322 & -2.197 & 0.623 & -0.399 & 47.917 & 0.427 \\
\hline ST5ST1 & 8.759 & -0.746 & -6.382 & 2.741 & 1.869 & -0.228 & 42.834 & 0.152 \\
\hline ST6ST1 & 8.759 & -10.350 & 61.886 & -0.125 & -3.384 & 0.961 & 39.530 & 0.588 \\
\hline ST7ST1 & 9.377 & 6.969 & -26.557 & 7.399 & 1.575 & -0.758 & 30.148 & 0.607 \\
\hline ST8ST1 & -2.068 & -6.234 & 26.943 & 3.670 & -3.442 & -0.851 & 30.417 & 0.649 \\
\hline ST9ST1 & -3.917 & 15.336 & 5.708 & 6.006 & -5.699 & 0.538 & 25.373 & 0.477 \\
\hline ST10ST1 & -14.148 & 18.789 & 0.998 & 6.709 & -5.029 & -0.019 & 26.288 & 0.489 \\
\hline Average & 0.626 & 1.161 & 17.817 & 2.642 & -1.829 & -0.132 & 36.522 & 0.423 \\
\hline
\end{tabular}

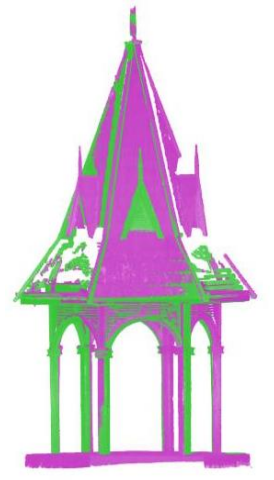

(a)

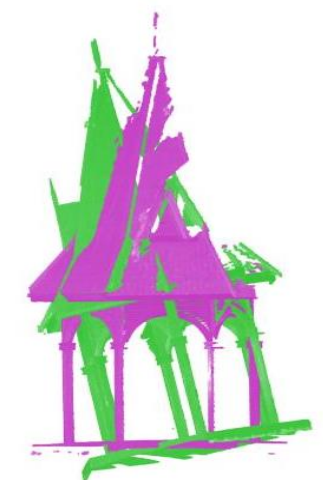

(b)

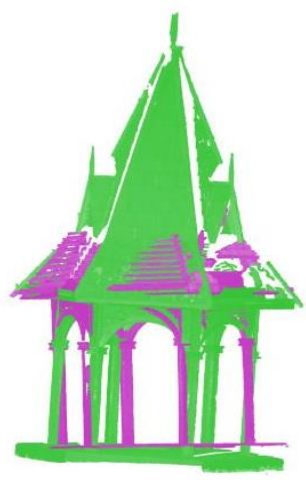

(c)

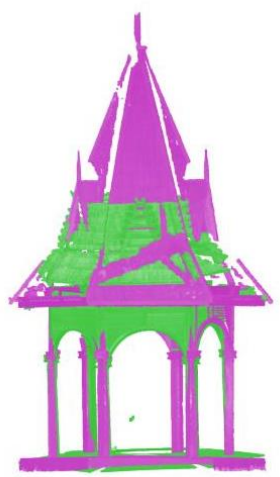

(d)

Figure 5. Pairwise coarse registration of (a) ST1-ST3 with lowest RMSE, (c) ST4-ST1 with large overlapping points, (b) ST8-ST1 with highest RMSE, and (d) ST1-ST9 with smallest overlapping.

shows the information about angular rotation and distance from source to target point cloud in order to align together. Study also shows that the distance between two point cloud affect the registration process (Weinmann, Weinmann, Hinz, \& Jutzi, 2011). In conclusion, small distance are required to avoid large propagation of point cloud that can cause to higher distance error.

\subsection{Data set II (Tawau Lighthouse)}

Tinagat Lighthouse is another one century preserved building exist since 1916 located on an uneven surface of Mount Tinagat. Its takes about 20 minutes from centre of Tawau to reach to this building. This building was built by Chance Brothers and Company, a company based in Smethwick, West
Midlands, United Kingdom. Its function is to provide the direction for ships during night (Ali, 2016). Fig. 6 and Fig. 7 show the actual structure of Tawau Lighthouse and the scan station locations respectively.

This historical building is cylindrical shape which give more challenge due to high degree of symmetry. This data set II provide us with lower overlapping point which is around $\approx$ $30 \%$. The characteristic of white colour of this building effect the quality of scans data as the TLS measurement principle influence by light reflection (Soudarissanane, Lindenbergh, Menenti, \& Teunissen, 2011). Nine stations is studied for outdoor scene in this data set under consideration of ability to merge the point clouds together. 
Table 4 shows the result for data set II. The reference station ST6 was randomly chosen to register all station unlike data set I that was using the first station. This is due close distance of ST6 to the others station permits roughly aligned for all the point cloud (Broersen, Peters, \& Ledoux, 2017).Aligning using all station to one reference station by means many-to-one relationship give lower average squared distance for this data but may not suggest a success registration.

Coarse registration preference more on high reliability of point between two point cloud for alignment (Pascal Willy Theiler, Wegner, \& Schindler, 2015). This was proven by the registration success of ST6-ST8 with lowest overlapping point as shown in Fig.8 (a). Plus, due to relative orientation that are approximate to identity matrix did contribute to success of registration (Pavan \& Dos Santos, 2017). The registration ST8ST6 seem well align but not at the base part of the Lighthouse as shown in Fig.8 (b). Moreover, highest overlapping point ST2-ST6 as can be seen in Fig. 9 (a) produces less well registered compared to alternate scan pair ST6-ST2 as in Fig. 9 (b) where the body of the building are well aligned but required further fine alignment to exact match to small part of the lightning rod due to symmetrical issue. Hence, the error distance used to define the accuracy also contributes to probability of corresponding point in two overlapping point cloud.

Good feature selection are essential factor to get high chance of corresponding point pairs matching. Like this case, the success registration occur at smallest overlapping point but the algorithm still managed to find the best matching to register the pairs. High degree of symmetry case which commonly found in building structure, with combination of using appropriate type of keypoint has able to reduce failure in registration (Yang, Dong, Liang, \& Liu, 2016). Here, the registration error occurs at the lightning rod part due to less data compared to overall surface of the lighthouse body. Average degree of rotation angle in this data are higher compared to data set I thus shows the higher propagation required by algorithm to do the aligning. The average results of transformation parameter also shows lower accuracy due to distance between two points are far compare to better alignment of previous data. Therefore, 3D Harris keypoint able to act as the suitable keypoint method in dealing with building structure data.

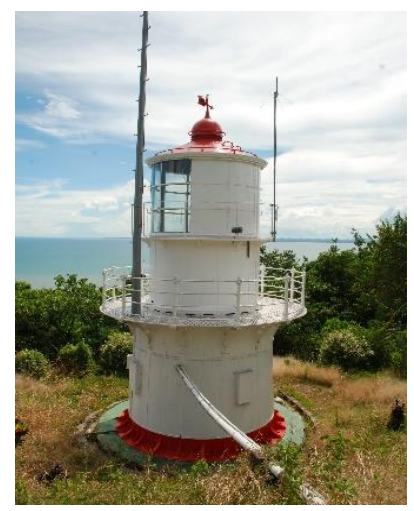

Figure 6.Tawau Lighthouse

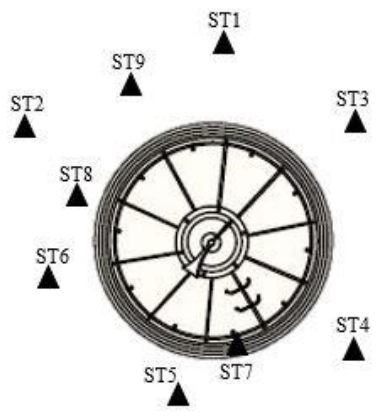

Figure 7. Scan location of Tawau Lighthouse.

Table 4. Scan pairs with transformation parameter, overlapping points and Root Means Square Error (RMSE). Results for data set II using point-to-plane minimization error and $0.05 \mathrm{~m}$ grid average downsampling method.

\begin{tabular}{ccccccccc}
\hline Scan pairs & $\omega\left(^{\circ}\right)$ & $\varphi\left(^{\circ}\right)$ & $\kappa\left(^{\circ}\right)$ & $\mathrm{Tx}(\mathrm{m})$ & $\mathrm{Ty}(\mathrm{m})$ & $\mathrm{Tz}(\mathrm{m})$ & Overlap $(\%)$ & RMSE $(\mathrm{m})$ \\
\hline ST6-ST1 & -1.554 & 1.416 & -75.044 & 4.808 & -4.194 & 4.200 & 35.884 & 0.808 \\
ST6-ST2 & -1.225 & -2.350 & -84.048 & 3.512 & -2.052 & 3.014 & 33.200 & 0.904 \\
ST6-ST3 & -3.291 & 0.816 & 30.062 & 0.310 & -4.093 & 1.387 & 33.673 & 0.427 \\
ST6-ST7 & -1.813 & -3.506 & 6.558 & -2.173 & 1.325 & -0.088 & 31.809 & 0.543 \\
ST6-ST8 & -1.622 & 1.039 & 23.899 & -1.058 & 0.151 & 0.311 & 23.343 & 0.081 \\
ST6-ST19 & -9.894 & 16.397 & -26.380 & -12.404 & 1.982 & 0.880 & 36.286 & 0.753 \\
& & & & & & & & \\
Average & -3.233 & 2.302 & -20.826 & -1.168 & -1.147 & 1.618 & 32.366 & 0.586 \\
& & & & & & & & \\
ST1-ST6 & 1.740 & 11.698 & 56.602 & 4.368 & 5.931 & -1.055 & 40.739 & 0.721 \\
ST2-ST6 & -22.361 & -7.755 & -87.155 & -5.514 & -9.368 & -1.610 & 44.115 & 0.625 \\
ST3-ST6 & 2.802 & -3.919 & 8.398 & -3.827 & 0.033 & -1.807 & 31.904 & 0.230 \\
ST7-ST6 & -0.297 & -6.800 & 23.311 & 1.046 & -2.312 & 0.024 & 26.958 & 0.402 \\
ST8-ST6 & 5.962 & -0.100 & -36.964 & 1.098 & 1.440 & -0.389 & 24.689 & 0.395 \\
ST19-ST6 & -3.432 & -0.716 & -32.738 & 5.381 & -3.114 & -4.158 & 27.276 & 0.588 \\
& & & & & & & & \\
Average & -2.598 & -1.265 & -11.425 & 0.426 & -1.232 & -1.499 & 32.613 & 0.493 \\
\hline
\end{tabular}




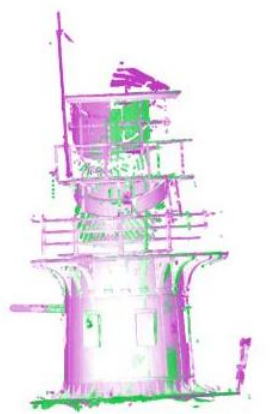

(a)

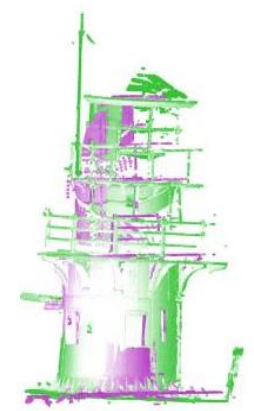

(b)
Figure 8. Pairwise coarse registration (a) for ST6-ST8, success register at smallest overlapping point and (b) ST8ST6 less well-registered.

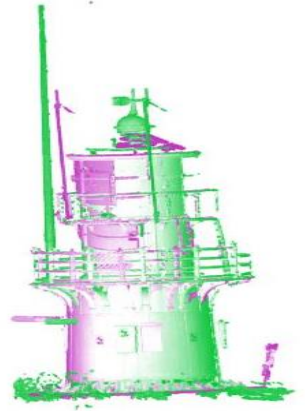

(a)

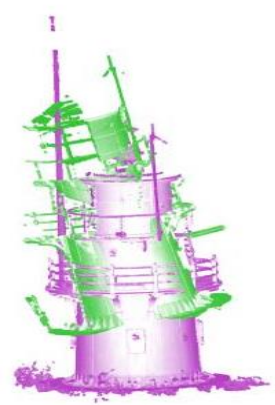

(b)
Figure 9. Pairwise coarse registration of (a) ST6-ST2 with highest RMSE due to miss matching at lighning rod part and (b) ST2-ST6 with less distace error and larger overlapping points.

\section{CONCLUSIONS AND FUTURE WORK}

Relative orientation based coarse pairwise registration have been studied in this paper. With the proper algorithm, it able to produce a success registration with low overlapping point cloud without help from artificial marker. Relative orientation also deals with evaluation on transformation parameters. The outcomes are well-registered when the distance between two point clouds are not too far from each other. Suitable selection of keypoint yield high corresponding point and managed to register point cloud with low overlapping percentage of points.

Moreover, the transformation matrix approximate to identity matrix can consider as a good selection for registration. Therefore, approaches in this paper is managed to register high geometrical symmetry of point cloud data.

In the future, the plan to further investigate is regarding as below details:

- $\quad$ Study on different dominant feature of 3D keypoint based on the characteristic of point cloud.

- Implementation of other algorithm for coarse and fine registration for better accuracy.

- $\quad$ Perform relative orientation using large data with clutter scene for detail evaluation of the algorithms.

\section{ACKNOWLEDGMENTS}

The authors would like to acknowledge the support from the Fundamental Research Grant Scheme (FRGS) under the grant number of FRGS/1/2017/ICT04/UNIMAP/02/1 from the Ministry of Education Malaysia.

\section{REFERENCES}

Ali, A. M. (2016). 3D Terrestrial Laser Scanning For Sabah Historical Landmark Documentation And Virtual Tourism. Journal of Built Environment, Technology and Engineering, 1, 321-329.

Baik, A. (2017). From point cloud to Jeddah Heritage BIM Nasif Historical House - case study. Digital Applications in Archaeology and Cultural Heritage, 4, 1-18. https://doi.org/10.1016/j.daach.2017.02.001

Barnea, S., \& Filin, S. (2008). Keypoint based autonomous registration of terrestrial laser point-clouds. ISPRS Journal of Photogrammetry and Remote Sensing, 63, 19-35. https://doi.org/10.1016/j.isprsjprs.2007.05.005

Brenner, C., Dold, C., \& Ripperda, N. (2008). Coarse orientation of terrestrial laser scans in urban environments. Photogrammetry \& Remote Sensing, 63, 4-18. https://doi.org/10.1016/j.isprsjprs.2007.05.002

Broersen, T., Peters, R., \& Ledoux, H. (2017). Automatic identification of watercourses in flat and engineered landscapes by computing the skeleton of a LiDAR point cloud. Computers and Geosciences, 106, 171-180. https://doi.org/10.1016/j.cageo.2017.06.003

Bueno, M., Martínez-Śanchez, J., Gonźalez-Jorge, H., \& Lorenzo, H. (2016). Detection of geometric keypoints and its application to point cloud coarse registration. International Archives of the Photogrammetry, Remote Sensing and Spatial Information Sciences - ISPRS Archives, 41, 187-194. https://doi.org/10.5194/isprsarchives-XLI-B3-187-2016

Che, E., \& Olsen, M. J. (2018). Multi-scan segmentation of terrestrial laser scanning data based on normal variation analysis. ISPRS Journal of Photogrammetry and Remote Sensing, 143, 233-248. https://doi.org/10.1016/j.isprsjprs.2018.01.019

Corina Daniela Păun, Valeria Ersilia Oniga, P. I. D. (2017). Three-dimensional transformation of coordinate systems using three-dimensional transformation of coordinate systems. International Journal of Engineering Science \& Reasearch Technology, 6(2), 355-363. https://doi.org/10.5281/zenodo.291839

Du, S., Zhang, Y., Zou, Z., Xu, S., He, X., \& Chen, S. (2017). Automatic building extraction from LiDAR data fusion of point and grid-based features. ISPRS Journal of Photogrammetry and Remote Sensing, 130, 294-307. https://doi.org/10.1016/j.isprsjprs.2017.06.005

Grant, D., Bethel, J., \& Crawford, M. (2012). Point-to-plane registration of terrestrial laser scans. ISPRS Journal of Photogrammetry and Remote Sensing, 72, 16-26. https://doi.org/10.1016/j.isprsjprs.2012.05.007 
Hansen, W. Von. (2004). Robust automatic marker-free registration of terrestrial scan data. In Photogrammetric Computer Vision (pp. 105-110).

He, Y., Liang, B., Yang, J., Li, S., \& He, J. (2017). An iterative closest points algorithm for registration of 3D laser scanner point clouds with geometric features. Sensors, 17(1862). https://doi.org/10.3390/s17081862

Klapa, P., Mitka, B., \& Zygmunt, M. (2017). Application of Integrated Photogrammetric and Terrestrial Laser Scanning Data to Cultural Heritage Surveying. IOP Conference Series: Earth and Environmental Science, 95(3), 1755-1315. https://doi.org/10.1088/1755-1315/95/3/032007

Kobbelt, L., \& Botsch, M. (2004). A survey of point-based techniques in computer graphics. Computers and Graphics, 28, 801-814. https://doi.org/10.1016/j.cag.2004.08.009

Li, M., \& Chen, Y. (2018). Registration of Laser Scanning Point Clouds: A Review. Sensors, 18(1641). https://doi.org/10.3390/s18051641

Maiseli, B., Gu, Y., \& Gao, H. (2017). Recent developments and trends in point set registration methods. Journal of Visual Communication and Image Representation, 46, 95-106. https://doi.org/10.1016/j.jvcir.2017.03.012

Pavan, N. L., \& Dos Santos, D. R. (2017). A global closed-form refinement for consistent TLS data registration. IEEE Geoscience and Remote Sensing Letters, 14(7), 1131-1135. https://doi.org/10.1109/LGRS.2017.2699940

Persad, R. A., \& Armenakis, C. (2017). Automatic 3D Surface Co-Registration Using Keypoint Matching. Photogrammetric Engineering \& Remote Sensing, 83(2), 137-151. https://doi.org/10.14358/PERS.83.2.137

Rusu, R. B., Blodow, N., \& Beetz, M. (2009). Fast Point Feature Histograms ( FPFH ) for 3D Registration. In IEEE International Conference on Robotics and Automation (pp. 3212-3217).

Rusu, R. B., \& Cousins, S. (2011). 3D is here: Point Cloud Library ( PCL ). In In: IEEE International Conference on Robotics and Automation (ICRA). IEEE, (pp. 1-4).

Salvi, J., Matabosch, C., Fofi, D., \& Forest, J. (2007). A review of recent range image registration methods with accuracy evaluation. Image and Vision Computing, 25, 578-596. https://doi.org/10.1016/j.imavis.2006.05.012

Shukor, S. A. A., \& Aminuddin, M. Q. (2017). Managing Huge Point Cloud Data through Geometrical-Based Registration. In Proceedings of the Second International Conference on Internet of things, Data and Cloud Computing (Vol. 166). https://doi.org/10.1145/3018896.3056783

Sipiran, I., \& Bustos, B. (2011). Harris 3D : a robust extension of the Harris operator for interest point detection on 3D meshes. Computer Vision, 27, 963-976. https://doi.org/10.1007/s00371011-0610-y

Soudarissanane, S., Lindenbergh, R., Menenti, M., \& Teunissen, P. (2011). Scanning geometry: Influencing factor on the quality of terrestrial laser scanning points. ISPRS Journal of
Photogrammetry and Remote Sensing, 66, 389-399. https://doi.org/10.1016/j.isprsjprs.2011.01.005

Theiler, P W, Wegner, J. D., \& Schindler, K. (2013). Markerless point cloud registration with keypoint-based 4points congruent sets. ISPRS Annals of Photogrammetry, Remote Sensing and Spatial Information Sciences, 2(5), 283288.

Theiler, P W, Wegner, J. D., \& Schindler, K. (2014). Fast registration of laser scans with 4-points congruent sets - what works and what doesn' t. ISPRS Ann. Photogramm. Remote Sens. Spatial Inf. Sci., 2(3), 149-156.

Theiler, Pascal Willy, Wegner, J. D., \& Schindler, K. (2014). Keypoint-based 4-Points Congruent Sets - Automated markerless registration of laser scans. ISPRS Journal of Photogrammetry and Remote Sensing, 96, 149-163. https://doi.org/10.1016/j.isprsjprs.2014.06.015

Theiler, Pascal Willy, Wegner, J. D., \& Schindler, K. (2015). Globally consistent registration of terrestrial laser scans via graph optimization. ISPRS Journal of Photogrammetry and Remote Sensing, 109, 126-138. https://doi.org/10.1016/j.isprsjprs.2015.08.007

Tremblay, J., \& Béland, M. (2018). Towards operational marker-free registration of terrestrial lidar data in forests. ISPRS Journal of Photogrammetry and Remote Sensing, 146, 430-435. https://doi.org/10.1016/j.isprsjprs.2018.10.011

Wang, Z., \& Claus, B. (2008). Point based registration of terrestrial laser data using intensity and geometry features. IAPRS and SIS, 33(B5), 583-589. https://doi.org/10.1.1.150.6063

Weinmann, M., Weinmann, M., Hinz, S., \& Jutzi, B. (2011). Fast and automatic image-based registration of TLS data. ISPRS Journal of Photogrammetry and Remote Sensing, 66, S62-S70. https://doi.org/10.1016/j.isprsjprs.2011.09.010

Xu, Y., Boerner, R., Yao, W., Hoegner, L., \& Stilla, U. (2019). Pairwise coarse registration of point clouds in urban scenes using voxel- based 4-planes congruent sets. ISPRS Journal of Photogrammetry and Remote Sensing, 151, 106-123. https://doi.org/10.1016/j.isprsjprs.2019.02.015

Yamany, S. M., Farag, A. A., \& Member, S. (2002). Surface signatures: An orientation independent free-form surface representation scheme for the purpose of objects registration and matching. IEEE Transactions On Pattern Analysis And Machine Intelligence, 24(8), 1105-1120.

Yang, B., Dong, Z., Liang, F., \& Liu, Y. (2016). Automatic registration of large-scale urban scene point clouds based on semantic feature points. ISPRS Journal of Photogrammetry and Remote Sensing, 113, 43-58. https://doi.org/10.1016/j.isprsjprs.2015.12.00

Revised August 2019 